form of slow and sharp waves, particularly in the frontal lobe, but also in the parietal and temporal lobes. Motor deficits were more marked on the contralateral side of the EFG abnormality. (Agarwal KN et al. Soft neurological signs and EEG pattern in rural malnourished children. Act a Paediatr Scand Nov 1989; 78:873-878).

COMMENT. The same authors have shown that malnourished children are at risk for depressed cognitive function, learning disabilities, and poor achievement at school. In the present paper they emphasize the occurrence of soft neurologic signs and EFG abnormalities in relation to poor nutrition. The influence of early malnutrition on subsequent behavioral development, learning disabilities, and soft neurologic signs has also been stressed by other authors (Galler JR et al. Pediatr Res 1984; 18:309 and 826). Perhaps the term minimal brain dysfunction (MBD) in relation to hyperactive children with learning disabilities was discarded prematurely in favor of ADHD. The pediatric neurologists role in the diagnosis and treatment of children with behavior and learning disabilities has been usurped to same extent by the increasing interests of the psychologists and developmental pediatricians. The importance of pediatric neurology and the recognition of symptams and signs of brain dysfunction in these patients should receive more emphasis, and nutrition and diet in nervous system disorders of children is a field for further investigation (Millichap JG. Nutrition, Diet, and Child Behavior. Charles C. Thamas, Springfield 1986).

\title{
UTILIZATION BEHAVIOR AND FRONTAL LOBE LESIONS
}

Utilization behavior was investigated in an adult with an acute behavioral disturbance, memory deficits, and a localized inferior medial bifrontal lesion at the Psychology Department, National Hospital, Queen Square, London; the MRC Applied Psychology Unit, Cambridge; and Department of Neurology, Atkinson Morley's Hospital, Wimbledon; and St. Andrews Hospital, Northampton, UK. Incidental utilization behavior was observed and categorized: 1) Toying, an object manipulated but not in a purposeful way (e.g. picking up a pencil but not using it for any purpose), 2) complex toying, two objects used in a linked way but in an incomplete fashion (e.g. picking up a pencil and using it to move objects), 3) coherent activity, set of actions integrated in a typical fashion (e.g. picking up a pen and paper and writing; picking up a pack of cards and dealing). Utilization behavior occurred when the patient was in conversation with the examiner and also when performing both verbal and nonverbal neuropsychological tests. His WAIS Verbal IQ was 73 and Performance IQ 79. Performance on spatial, perceptual, language, and simple praxic tests was satisfactory in contrast to tasks involving a frontal or long term memory component which were uniformly and severely impaired. The utilization behavior was present in the absence of confusion or dementia. The utilization behavior occurred most frequently in the brief intervals between tasks, and more often when auditory verbal rather than visual motor tasks were being performed. A differentiation 
was made between two forms of utilization behavior: 1) an incidental form, as exhibited by the patient; and 2) an induced form, occurring with Lhermitte's procedure where the examiner stimulates the palm and fingers of the patient's hands with the object. (Shallice T et al. The origins of utilization behavior. Brain Dec 1989; 112:1587-1598).

COMENT. Utilization behavior investigated in this adult patient might also be evaluated in children with learning and memory disorders and may assist in the neuroanatomical localization of lesions in the frontal lobe. Lhermitte's neuroanatamical account of utilization behavior is based on the theories of Denny-Brown and a possible imbalance between the activities of frontal and parietal lobes. Visual stimuli activate parietal lobe systems which in turn initiate actions normally inhibited by frontal lobe systems. Damage to the frontal lobes leading to unmodulated effects of parietal systems may result in utilization behavior. Children with minimal brain dysfunction and hyperactivity have an increased tendency to touch and toy with articles within their reach.

\section{METABOLIC DISOPDERS}

PERI PHERAL NEUROPATHY AND HEREDITARY TYROS INEMIA

Neurologic crises in 48 children with tyrosinemia identified on neonatal screening since 1970 are described from the Departments of Genetics, Hopital Sainte Justine, Hopital de Chicoutimi; and Universite Laval, Quebec, Canada. Neurologic crises had occurred in $20(42 \%)$ and began at a mean age of one year leading to 104 hospital admissions. Abrupt episodes of peripheral neuropathy were characterized by severe pain with extensor hypertonia (75\%), vomiting or paralytic ileus $(69 \%)$, muscle weakness $(29 \%)$, and self-mutilation $(8 \%)$. Fourteen patients died due to complications of respiratory insufficiency or mechanical ventilation. In 5 patients undergoing hepatic transplantation none had neurologic crises. Urinary excretion of gamma aminolevulinic acid, an intermediate of porphyrin biosynthesis, was elevated during crises and during asymptamatic periods. Axonal degeneration and demyelination were demonstrated by EMG, NCS and neuromuscular biopsies. (Mitchell G et al. Neurologic crises in hereditary tyrosinemia. N Engl $J$ Med Feb 15, 1990; $322: 432-437$ ).

COMMENT. This study indicates that episodes of acute severe peripheral neuropathy are common in hereditary tyrosinemia and resemble crises of the neuropathic porphyrias. Hepatic transplantation eliminated the neurologic crisis, the major cause of mortality in these patients.

Abnormal porphyrin metabolism appears to underlie the neurologic crisis of tyrosinemia and is associated with elevated gamma aminolevulinic acid excretion. Therapy for the neurologic crises 